\title{
Les transparences de la médiation
}

Anthropologie d'un patrimoine hors l'histoire

Transparencies of mediation. The anthropology of heritage outside history

\section{Claudie Voisenat}

\section{CpenEdition}

\section{Journals}

Édition électronique

URL : https://journals.openedition.org/insituarss/469

DOI : $10.4000 /$ insituarss.469

ISSN : 2680-4972

Éditeur

Ministère de la Culture

Référence électronique

Claudie Voisenat, «Les transparences de la médiation », In Situ. Au regard des sciences sociales [En

ligne], 1 | 2019, mis en ligne le 15 octobre 2019, consulté le 19 décembre 2022. URL : http://

journals.openedition.org/insituarss/469; DOI : https://doi.org/10.4000/insituarss.469

Ce document a été généré automatiquement le 19 décembre 2022.

\section{(c) () 8 (}

Creative Commons - Attribution - Pas d'Utilisation Commerciale - Pas de Modification 4.0 International - CC BY-NC-ND 4.0

https://creativecommons.org/licenses/by-nc-nd/4.0/ 


\title{
Les transparences de la médiation
}

\author{
Anthropologie d'un patrimoine hors l'histoire \\ Transparencies of mediation. The anthropology of heritage outside history
}

\section{Claudie Voisenat}

1 Églises aux caractéristiques inhabituelles, demeures alchimiques, hauts lieux vibratoires, nœuds d'énergie cosmo-tellurique..., le patrimoine est parfois l'objet de curieuses visites reposant sur la double conviction que le site a un sens caché, dépassant largement son intérêt historique ou artistique, et que la découverte de ce que le lieu révèle amène à en avoir une véritable connaissance qui, loin d'un simple savoir, s'assimile à une expérience initiatique, susceptible de transformer celui qui la vit. De la cathédrale à l'ermitage de campagne, du château cathare au site mégalithique, des thermes gallo-romains aux abris sous roche, ces sites, très différents par leur nature, leur taille ou leur notoriété sont cependant tous supposés entretenir un certain rapport avec le « sacré » et ne sauraient être abordés, selon les médiateurs de ces usages inédits du patrimoine, à la seule lumière de la raison ou être considérés comme de simples supports de savoirs. Bénéficier du pouvoir de ces lieux où l'homme se sent « relié » au tout de l'univers impose de renoncer à son "mental ", d'écouter son "moi intérieur ", de «lâcher prise». Toutes ces postures nécessitent un apprentissage - d'ailleurs souvent mis en scène par ces visites singulières.

2 Certes, le discours sur le patrimoine, et plus largement sur les œuvres d'art, oscille depuis toujours entre érudition et émotion. Cette tension semble constitutive de l'idée même d'un patrimoine pensé comme témoin de l'histoire. Mais le curseur n'est-il pas aujourd'hui en train de se déplacer très significativement du côté de ce qu'on appelle le "ressenti», privilégiant l'expérience individuelle, le dialogue intérieur avec le monument, au détriment de formes plus traditionnelles de communication d'un savoir lié à l'histoire de l'art? Les médiations auxquelles certains sites patrimoniaux donnent lieu seraient-elles en train de devenir une des nombreuses techniques d'empowerment par lesquelles les individus sont aujourd'hui sommés de se prendre en charge, de devenir maîtres de leur destin et de transformer ce qu'ils reçoivent en capacité d'action? Bref, le patrimoine, habituellement considéré comme bien collectif par 
excellence, comme support d'un discours sur l'histoire nationale, serait-il en passe de se transformer en instrument de développement personnel ?

3 Telles sont les questions amenées par l'observation et l'analyse - l'ethnographie en un mot - des médiations du patrimoine organisées autour d'un des fleurons du patrimoine religieux français, aujourd'hui plutôt considéré comme un haut lieu de spiritualité : la basilique Sainte-Marie-Madeleine de Vézelay, située au cœur de la Bourgogne, dans le département de l'Yonne, et inscrite depuis 1979 au Patrimoine mondial de l'humanité.

Sur le chemin de Vézelay.

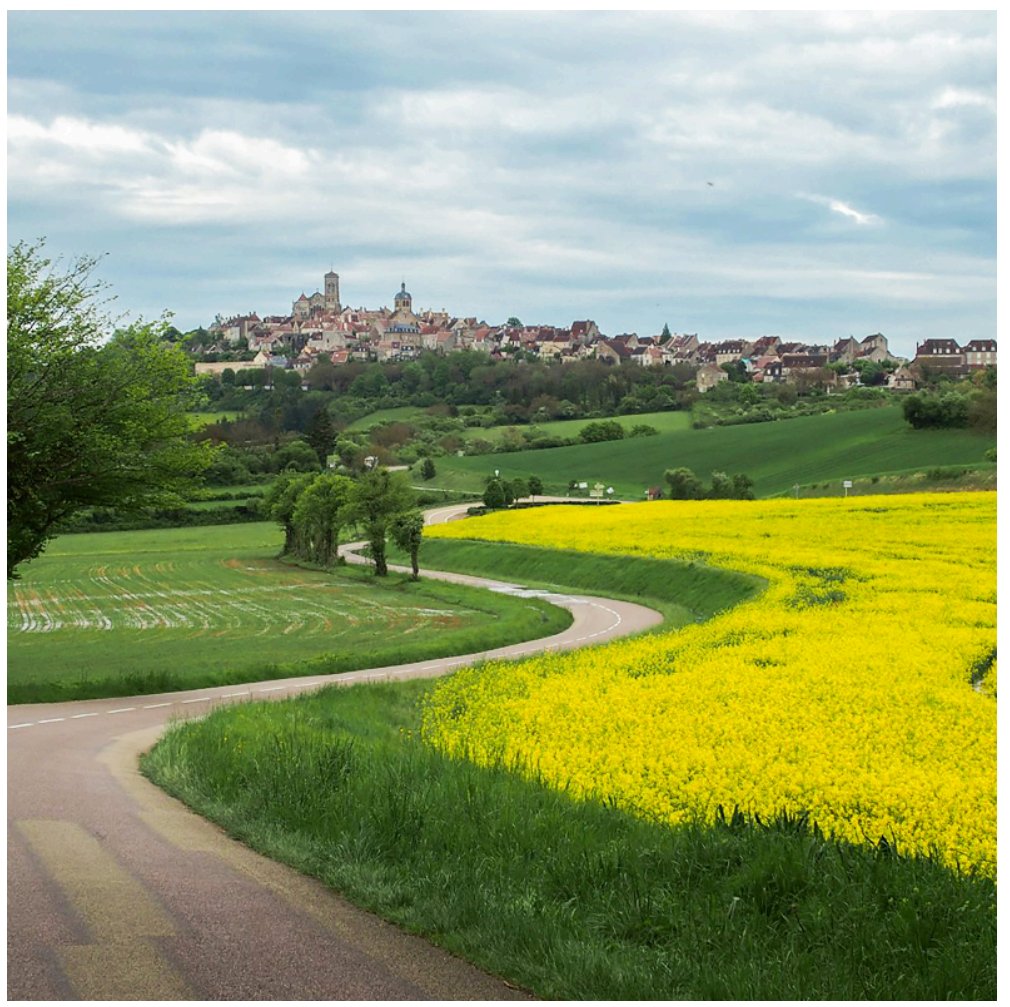

«Visible des quatre horizons », la colline de Vézelay - ou colline du Scorpion d'après son nom romain, pour les férus d'ésotérisme.

(c) Marie Adamski.

\section{L'esprit du lieu}

La basilique de Vézelay présente un certain nombre de caractéristiques qui en font un lieu d'exception. Sa situation d'abord. "Visible des quatre horizons", elle est construite au sommet d'une colline sur laquelle les moines du IX ${ }^{e}$ siècle, lassés de voir leur abbaye détruite par les envahisseurs normands, étaient venus se réfugier. Construite ou reconstruite à différentes époques, entre 1120 et $1180^{1}$, le passage de l'art roman à l'art gothique est inscrit dans son architecture même, avec une nef romane, un chœur gothique et une avant-nef dont les arcs brisés annoncent la transition entre les deux styles. Les chapitres historiés de la nef et du narthex sont considérés comme typiques de l'art de la Bourgogne romane, tandis que le traitement architectural de la luminosité évoque une véritable dramaturgie, fondée sur une conception théologique et mystique de la lumière que l'on verra surtout à l'œuvre dans le gothique (voir Duby 
1976). Ici, cependant, le mélange des styles lui donne une configuration particulière : le chœur inondé de clarté succède à une nef plongée dans un doux clair-obscur; les projections lumineuses des fenêtres viennent éclairer les chapiteaux supérieurs du mur nord au solstice d'hiver, jouer sur les chapiteaux inférieurs à Pâques et déposer neuf taches de lumière sur la travée centrale de la nef au solstice d'été, formant un chemin lumineux de la pénombre du narthex vers l'illumination du chœur. On a ainsi pu dire que la lumière était le matériau principal de l'église et que celle-ci permettait d'expérimenter physiquement et spirituellement le passage de l'ombre à la lumière, c'est-à-dire le processus même de la conversion religieuse ${ }^{2}$.

L'appel de la lumière 1

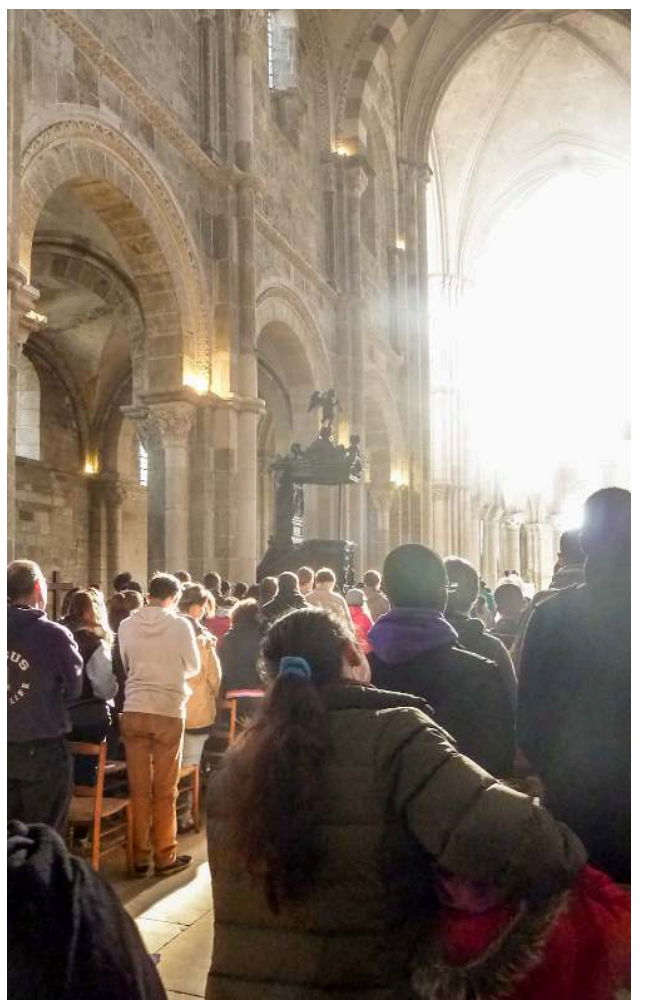

Le chœur illuminé prolonge la pénombre relative de la nef.

(c) Claudie Voisenat.

La basilique est aussi remarquable par la présence des reliques présumées de MarieMadeleine. C'est vers le milieu du XI ${ }^{e}$ siècle que le prieur de Vézelay fait courir le bruit qu'un moine y aurait rapporté le corps de la sainte pour le protéger des invasions sarrasines. Un pèlerinage s'organise et Vézelay devient un point de passage obligé sur la route de Saint-Jacques-de-Compostelle. L'abbatiale s'agrandit à mesure que croît sa renommée, et c'est de Vézelay que Bernard de Clairvaux lance en 1146 son appel à la deuxième croisade. Le déclin cependant n'allait pas tarder. En 1279, le pape reconnaît les reliques de Marie-Madeleine à Saint-Maximin, en Provence. Le pèlerinage de Vézelay est déserté, tandis que l'avènement des ordres mendiants vient changer les pratiques de dévotion. En 1562, lorsque Vézelay tombe aux mains des protestants, les reliques sont dispersées et la basilique transformée en écurie. Ce qui en subsiste aujourd'hui (essentiellement une côte) résulte d'un don fait à la fin du XIX ${ }^{e}$ siècle par l'archevêché de Sens qui l'avait reçue en cadeau en 1281. 
L'appel de la lumière 2

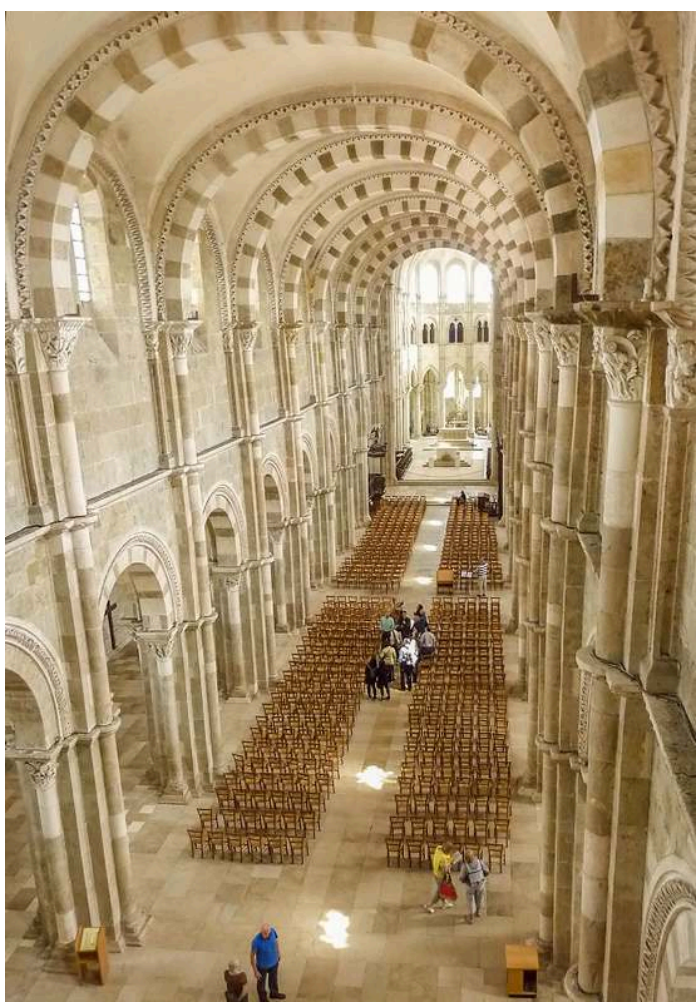

Au solstice d'été les neuf taches de lumière qui mènent de la nef vers le chœur.

(c) Claudie Voisenat.

6 Le $\mathrm{XX}^{\mathrm{e}}$ siècle achève de faire de Vézelay un haut lieu de rencontre entre art et spiritualité. Romain Rolland vient y finir sa vie. Paul Claudel y séjourne souvent pour y retrouver celle qui a inspiré le personnage d'Ysée dans Partage de midi et dont il a eu une fille. Le couple formé par Christian Zervos, critique et collectionneur d'art, et sa femme Yvonne, y reçoit Pablo Picasso, Paul Éluard et bien d'autres. Georges Bataille s'y installe, et après lui Max-Pol Fouchet, Maurice Clavel et Jules Roy, tous enterrés dans le petit cimetière en contrebas de la basilique.

De nos jours, le musée Zervos, installé dans la maison de Romain Rolland, présente les plus belles œuvres léguées par le collectionneur à la Ville de Vézelay, tandis que des expositions, des conférences, des résidences d'artistes ou d'écrivains, des concerts entretiennent la vie culturelle et tentent de promouvoir une vision du site moins religieuse et plus artistique, et que depuis quelques années un tourisme de la gastronomie et de la viticulture cherche également à se développer.

Mais le tourisme vézélien, qui draine près d'un million de visiteurs par an pour moins de cinq cents habitants permanents, demeure essentiellement centré sur la basilique, favorisé par l'implantation en 1993 de la Fraternité monastique de Jérusalem, une jeune congrégation fondée en 1975, également présente au Mont-Saint-Michel, chargée de l'« animation liturgique, spirituelle et culturelle de la basilique ${ }^{3}$ et qui développe une vision esthétique de la religion. Parallèlement, comme le souligne le site des amis de Vézelay, «des initiatives syncrétiques ou plus ou moins ésotériques bénéficient de l'attrait du lieu $»^{4}$. 


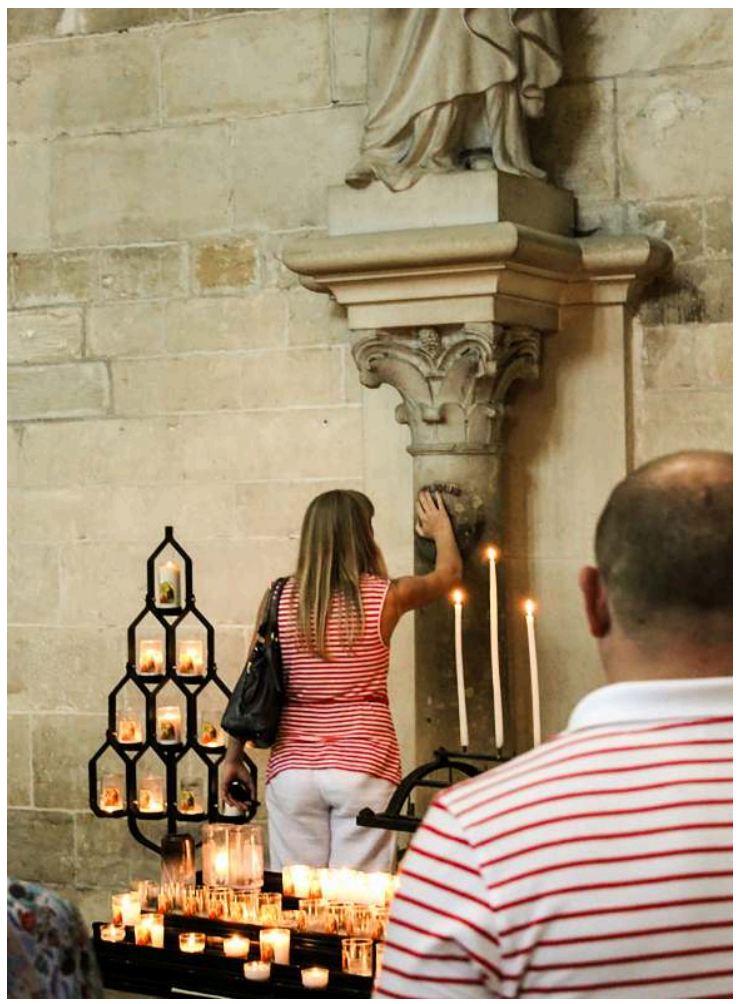

La colonne reliquaire contient des fragments de relique de la Madeleine de Saint-Maximin en Provence, recueillies et apportées à Vézelay dans les années 1920. Ce qu'il reste aujourd'hui des reliques proprement vézeliennes (essentiellement une côte) est contenu dans une châsse placée dans la crypte. Leur authenticité a été mise en cause par le pape dès le XIII siècle et l'abbatiale dépossédée du culte canonique.

(c) Jing Wang.

\section{Autour de la basilique}

Dans ce contexte, je me suis attachée à analyser les médiations mises en œuvre autour de la basilique ${ }^{5}$.

10 Tout d'abord, la confrérie monastique elle-même propose des visites toute l'année, les dimanches après-midi, et tous les jours aux mois de juillet et août. Les visites sont assurées soit par des moines ou des moniales, soit par des conférenciers privés auxquels la confrérie fait appel lorsqu'elle ne peut assurer les visites. C'est le cas par exemple d'une jeune femme, médiéviste et peintre d'enluminures, qui possède un atelier dans le village et propose par ailleurs des stages d'apprentissage de l'art d'enluminer.

11 Je me suis également intéressée aux médiations mises en œuvre par la Maison du visiteur, créée en 1994, située à mi-chemin de la rue qui monte vers la basilique, et dont la directrice artistique, diplômée de l'École du Louvre, se reconnaît dans une approche spirituelle et néo-platonicienne de l'art. La Maison du visiteur participe au réseau international Et si la beauté pouvait sauver le monde ${ }^{? 6}$, qui organise depuis 1993 des rencontres régulières sous forme de congrès (Barcelone en 2011, Québec en 2014, Paris en 2017). Ces rencontres sont l'occasion d'affirmer que le beau est un chemin vers la lumière, vers le divin, quelles que soient ses formes, qu'il est porteur de vie et de sens, 
agit sur l'homme et lui redonne dignité et espoir. Ce réseau a été créé par le comédien Olivier Fenoy, fondateur en 1963 de l'office culturel de Cluny (OCC), communauté mixte de laïcs d'obédience catholique souhaitant vivre leur foi au travers d'activités artistiques et culturelles, et qui se sont fortement impliqués dès les années 1970 dans l'animation socio-culturelle ${ }^{7}$. Leur pensée prône l'engagement mutuel dans un compagnonnage qui permet de faire de chacun un «sujet», selon le principe énoncé par saint Augustin qu'" une personne est achevée par une autre personne ». L'OCC se réclame du personnalisme d'Emmanuel Mounier, fondateur de la revue Esprit. La Maison du visiteur ne propose pas d'emblée une visite du monument lui-même mais un parcours scénographique à l'aide de films, de maquettes et de reconstitutions de chapiteaux, qui permettent de comprendre les «noces de la lumière et de la pierre » et le sens universel des "humbles messages» et "puissants enseignements» que les bâtisseurs «ont déposé[s] dans le silence de la pierre » et qui transforment chaque chapiteau en un «miroir de la vie des hommes ». La Maison ${ }^{8}$ propose non seulement des visites mais également des ateliers qui ouvrent à une expérience plus intime, à travers la marche, le dessin, l'expression orale...

La Maison du visiteur.
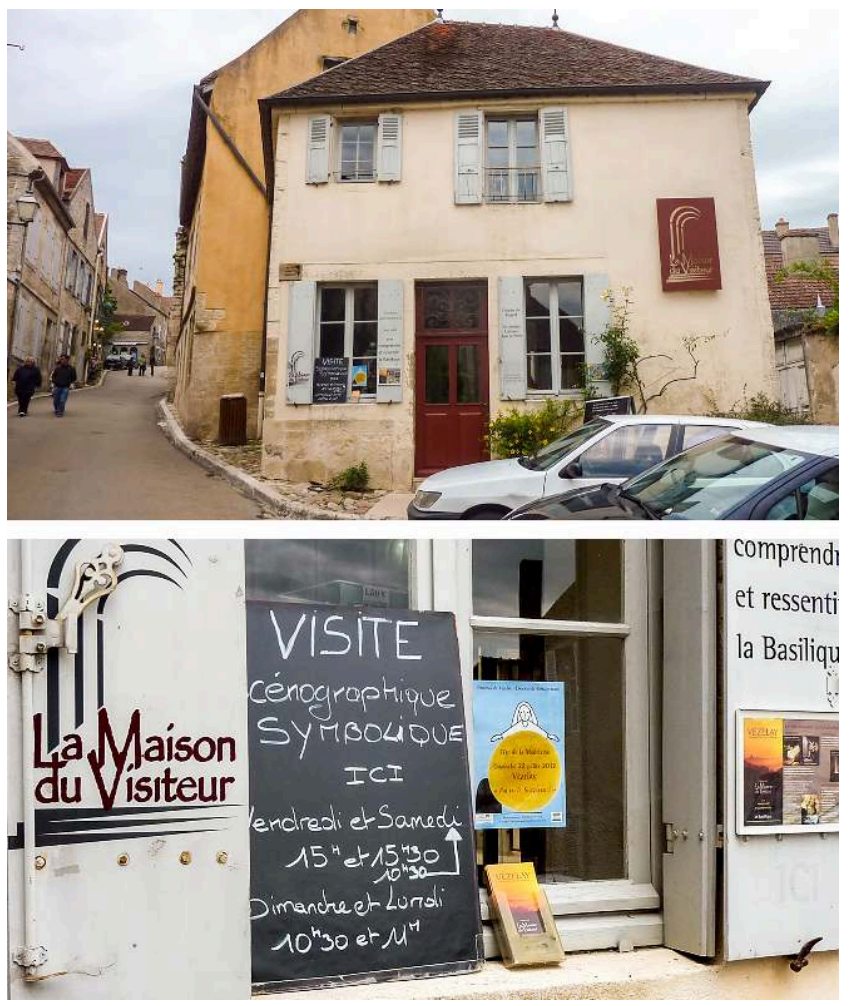

Située à mi pente de la rue qui mène à la basilique, la Maison du visiteur propose une sorte de pédagogie sensible préparatoire à la visite du monument. Elle choisit de délivrer les savoirs jugés indispensables à la "rencontre entre l'édifice et son visiteur », mettant en place les conditions d'un renversement à l'issue duquel c'est le visiteur qui est visité.

(c) Claudie Voisenat.

Enfin, je me suis attachée aux médiations de l'association Convergences, créée en 1992 par le propriétaire de la librairie ésotérique de Vézelay, L'Or des étoiles, qui venait d'ouvrir l'année précédente. Cette association nommée d'après la phrase de Teilhard de Chardin " Tout ce qui monte converge », se définit comme un " espace de liberté tourné 
vers la connaissance de l'humain ${ }^{9}$. Elle organise des concerts et des expositions, propose des rencontres, des conférences, des débats et des stages. Le propriétaire de la librairie est l'un des conférenciers agréés de la basilique et organise, sur demande, des visites de groupe. Il a également animé tous les ans jusqu'en 2014 un stage sur la géométrie et l'architecture sacrée, en lien avec deux autres intervenants, un géobiologue conseiller en bio-habitat (à l'intersection de la radiesthésie et du feng shui) et un spécialiste de la symbolique romane. Ils y proposent une lecture énergétique et cosmique du lieu et de son architecture qui est en même temps une forme d'introspection, dans la mesure où "accéder à la réalité subtile de la Madeleine de Vézelay, c'est d'abord s'ouvrir à soi-même ». Ce stage, auquel j'ai participé en 2012, dans le cadre d'une recherche sur les lectures ésotériques du patrimoine, a été le point de départ de mon intérêt pour les diverses médiations destinées à répondre aux demandes spirituelles des visiteurs et d'une comparaison avec les autres offres de médiations patrimoniales proposées à Vézelay ${ }^{10}$.

13 Si toutes ces médiations s'ancrent dans l'architecture du lieu et se réfèrent plus ou moins aux mêmes matériaux - les chapiteaux historiés, le grand tympan sculpté, les jeux de lumière et leur utilisation métaphorique dans le registre spirituel de la conversion ou de l'expérience transformative -, chacune n'en garde pas moins sa spécificité : lecture paradoxalement plus historique de la part de la congrégation, plus symbolique et contemplative pour la Maison du visiteur, plus énergétique et reliant l'homme à l'univers pour l'association Convergences ${ }^{11}$.

Il n'en reste pas moins que toutes partagent certaines caractéristiques que je voudrais maintenant expliciter rapidement, et qui proposent du patrimoine une vision sensiblement différente de celle que l'on voit à l'œuvre dans les médiations plus strictement historiques. J'en soulignerai ici deux, dont nous verrons qu'elles ne sont pas sans incidence sur les formes de médiation proposées : d'une part, le patrimoine est porteur d'un message pour celui qui se met à son écoute ; d'autre part, il est agissant, c'est-à-dire doté de pouvoirs spécifiques, susceptibles d'avoir des effets sur le visiteur, de le transformer: une "expérience transformatrice », susceptible de lui redonner la capacité d'agir, de créer, de reprendre en main sa propre vie. 

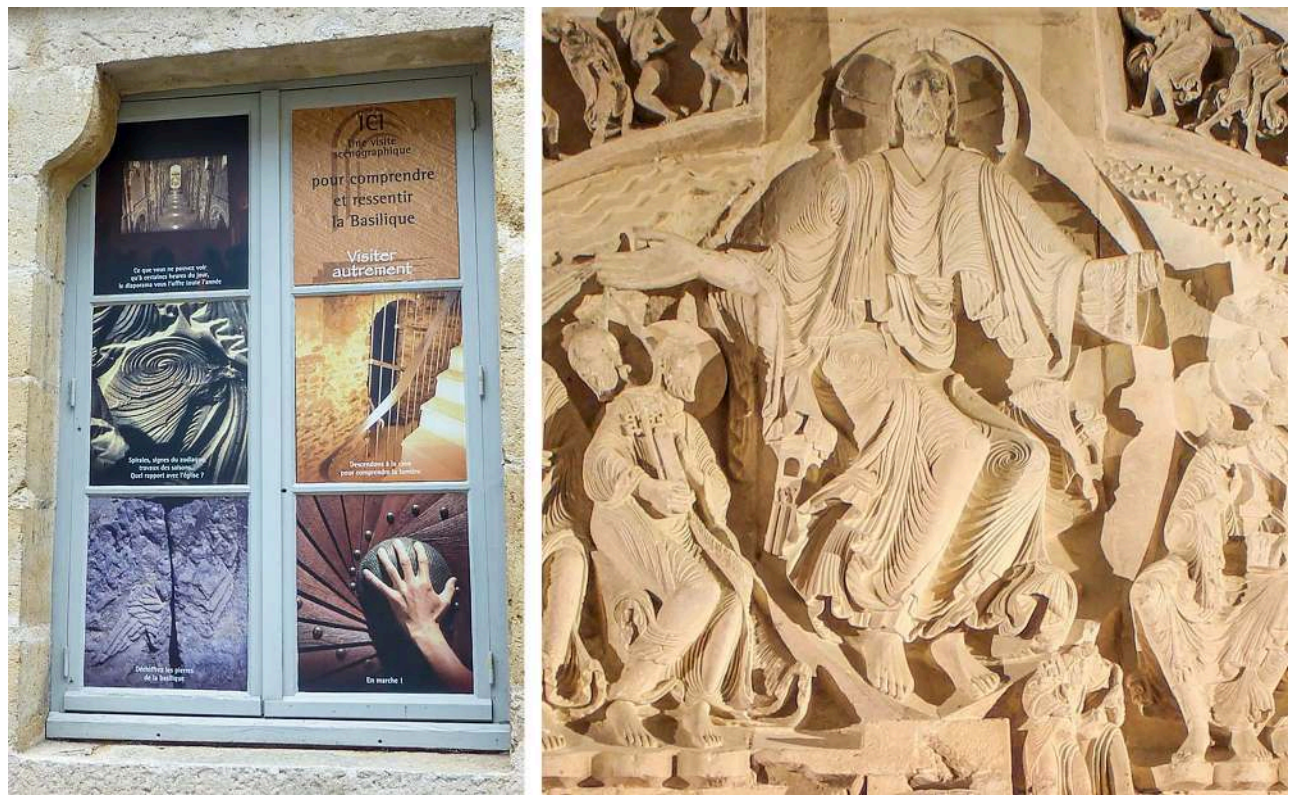

Les scénographies et les animations proposées par la Maison du visiteur sont ancrées dans une symbolique universelle du sacré où la lumière et les formes jouent un rôle de premier plan. Les spirales sur les genoux et les hanches du Christ du tympan de la basilique sont ainsi ramenées à la forme universelle, celle de la vie (depuis les escargots jusqu'aux galaxies) prouvant que tout, dans l'univers est relié, microcosme et macrocosme.

(c) gauche Claudie Voisenat / Office du tourisme Vézelay ; (c) droite Claudie Voisenat.

\section{À l'écoute de la dimension cachée}

Considérons le premier point. De façon tout à fait conventionnelle, la Congrégation considère le monument comme porteur d'un message d'espoir, celui de la résurrection, particulièrement représenté par le Christ du grand tympan répandant le Saint-Esprit sur ses disciples. À l'inverse de Mérimée, qui ne voyait dans les chapiteaux sculptés que la terreur que l'Église faisait régner par ses représentations de démons et de châtiments, on lit aujourd'hui dans les ondulations qui agitent les drapés des vêtements le souffle de l'esprit et dans l'orientation est-ouest du bâtiment le triomphe de la vie et de la lumière sur la mort et l'obscurité ${ }^{12}$.

À cette première lecture, la Maison du visiteur en superpose une autre : à contempler l'œuvre, on ne peut qu'admirer un art de bâtir qui, nous dit-on, « invite à réfléchir sur les organisations humaines, le sens du métier, la coresponsabilité, l'exercice de l'autorité et l'engagement commun comme forces de création et d'innovation $»^{13}$. Le monument est ici porteur d'un message de beauté, de cohérence et d'harmonie, celles de l'univers que les bâtisseurs se sont efforcés de retranscrire dans le microcosme de l'église. Le visiteur, en acceptant de recevoir cette beauté, se laissera rejoindre par le témoignage de cette société de marcheurs et de constructeurs, familière du mystère, imprégnée par les Écritures, portée par la foi et dotant le monde d'un sens que nous avons perdu et que notre société matérialiste a grand besoin de retrouver. Dans cette vision néo-platonicienne de l'esthétique, la contemplation du beau mène au bon et au vrai (cette « ancienne trinité », selon le mot de Dostoïevski), et le beau est ici universel, placé sous le signe de la lumière mais aussi de la spirale : celle que l'on retrouve dans 
les plis de la robe du Christ du grand tympan mais aussi, nous explique-t-on avant d'entrer dans les salles didactiques de la Maison du visiteur ${ }^{14}$, sur la coquille des escargots, dans les pommes de pin, dans l'implantation des cheveux au sommet de notre crâne et dans la forme des galaxies, comme une démonstration que tout, dans l'univers, est relié.

C'est aussi un message depuis longtemps perdu que le stage sur la géométrie et l'architecture sacrée invite à percevoir; et ce message, là encore, se superpose aux précédents sans les annuler, ni les contredire. Ce que les bâtisseurs ont cherché à nous transmettre, ce sont les savoirs de la "Tradition", des savoirs bien plus anciens que la religion chrétienne, des savoirs païens, celtiques, d'abord inscrits dans les mégalithes puis dans les pierres des cathédrales, dissimulés parmi la profusion d'images d'une religion qui cherchait à les éradiquer. Ces savoirs, énergétiques, cosmiques, permettent la rencontre entre la Terre et le Ciel, restaurent l'image d'une déesse-mère serpentine, vouivre, représentant les énergies de la terre, et désignent certains points particuliers comme des lieux de communication cosmo-telluriques, hauts lieux énergétiques sur lesquels les hommes se sont plu à dresser des pierres gigantesques puis à bâtir des lieux de culte. On reconnaît là, bien entendu, des motifs amplement déployés par les romans mystico-ésotérico-policiers contemporains tels le célébrissime Da Vinci Code et La Promesse de l'ange de Frédéric Lenoir et Violette Cabesos, qui a pour cadre le MontSaint-Michel.

\section{Jeux de ventriloquie : le patrimoine comme expérience transformatrice}

Le second point est un prolongement du premier. La caractéristique de ces messages inscrits dans le monument est en effet qu'ils sont agissants. La congrégation s'en tient au pouvoir de la métaphore lumineuse. Le pèlerin, nous explique-t-on, était jadis retenu dans la pénombre de l'avant-nef, où il pouvait parfois demeurer plusieurs jours, comme en un lieu de transition entre le monde extérieur et le sanctuaire, un espace de purification. "Confiné dans cet espace clos, le pèlerin se tourne bien sûr vers le grand portail : lorsque celui-ci s'ouvre, il ressent un véritable appel vers la lumière, vers plus de lumière... Jésus est "la porte", et lorsque celle-ci s'ouvre, il comprend qu'Il est bien "la lumière du monde". » La lumière agit donc comme un moteur, qui permet de se mettre en route, d'aller du narthex vers le chœur, d'ouest en est, de la mort à la résurrection.

Pour la Maison du visiteur, la rencontre avec le monument peut être l'occasion d'une véritable expérience transformative: "L'art roman s'adresse à tout l'homme, corps, cœur et esprit, par un chemin plus intuitif qu'explicatif : regard, écoute, sensation et émotion sont les véhicules d'une expérience qui opérait une transformation dans l'âme du pèlerin. Aujourd'hui, dans ces lieux sans ruptures [...], la rencontre de Vézelay peut être l'occasion d'un événement intérieur, d'un retournement ${ }^{15}$. Car le monument, nous dit-on, n'est pas seulement un lieu de passage vers l'intériorité de l'homme, c'est un «sanctuaire vivant ». Plus largement d'ailleurs, c'est tout le patrimoine qui, comme l'écrit la directrice artistique, est vivant ou, plus exactement, fonctionne comme un équivalent symbolique des peuples : «La blessure du patrimoine est profonde : ce sont les larmes versées sur le cœur de Beyrouth, de Bagdad, de Kaboul, de Lhassa... Ce sont les destructions des bouddhas, des icônes, des temples de prière. Ce sont les campagnes 
acharnées de négation des savoir-faire ancestraux au profit d'opportunismes aveugles. Ce sont les files des peuples en exode. La blessure est profonde parce qu'elle touche le corps tout entier des peuples, les laissant mutilés, sans formes et sans voix, livrés à la survie, parfois à la haine [...]. Loin d'être seulement une richesse à sauvegarder ou un site à visiter absolument, [le patrimoine] nous révèle qu'il est d'abord le corps et la figure sacrée des peuples, leurs vêtements de dignité. Face à toutes les formes contemporaines de mise à mort, il s'offre comme un geste culturel qui pose la question ultime de l'être $»^{16}$. Le patrimoine est donc ici non seulement agissant mais vivant, susceptible de s'adresser directement au visiteur, de le "visiter ", d'être finalement son propre médiateur, voire un médium permettant de recevoir les "messages" des bâtisseurs du passé, témoins parmi d'autres d'une sagesse universelle où l'homme n'est pas coupé du divin.

Comme la rencontre avec le monument, cette expérience transformatrice est mise en scène par des médiations qui la préparent puis l'explicitent. Une visite proposée à des jeunes d'un lycée agricole, venus accompagnés de leurs correspondants de divers continents (Europe, Asie et Amérique latine), en illustre parfaitement la trame. Après avoir regardé un film présentant des vues très esthétisées de la basilique sur fond de musique classique, les jeunes - et moi-même - sommes invités à prendre planche à dessin, feuilles et crayon, à cheminer jusqu'au monument, à y entrer et à nous $\mathrm{y}$ installer pour dessiner une vue ou un détail laissé à notre choix. Nous disposons pour cela de quarante-cinq minutes au terme desquelles nous devons revenir à la Maison du visiteur présenter notre travail. Je choisis quant à moi de dessiner une des ouvertures percées dans la partie supérieure de la nef pour l'éclairer. Piètre dessinatrice, je m'appliquais à tracer les grilles de fer qui barrent l'ouverture. De retour auprès de la directrice qui anime elle-même cet atelier, nous sommes conviés à prendre une autre feuille, à fermer les yeux, à faire "lâcher prise " à notre mental et à redessiner de mémoire - sans regarder - la vue que nous avions choisie. On imaginera sans peine que les dessins ainsi obtenus étaient pour le moins déstructurés. La phase suivante consista pour chacun d'entre nous à présenter les deux versions et à les soumettre au commentaire de la médiatrice. Celui-ci mobilisait, au cas par cas, un double argumentaire : d'une part, la liberté acquise une fois abandonné le carcan du mental et de la maîtrise de soi; de l'autre, l'interprétation des lignes déstructurées du dessin comme un jaillissement de lumière, un rayonnement, une révélation, celle de la vérité intérieure, débarrassée du carcan des normes de l'esthétique et de la rationalité. Les jeunes se révélèrent fort impressionnés par la démonstration, dont le caractère œcuménique, fondé sur une symbolique de la lumière, lui assurait d'être comprise par tous les participants, qu'ils soient croyants ou non, et quelle que soit leur religion. De fait, tous sont repartis avec le sentiment d'avoir vécu une expérience particulière qui n'allait peut-être pas bouleverser leur vie, mais qui allait - ils en étaient sûrs - enrichir leur vision du monde. Ici, la médiation provoque puis explicite les conditions d'une rencontre directe entre le monument et ses visiteurs, d'un dialogue intime qui passe par la double médiation du dessin puis de l'explication. Deux médiations qui demeurent cependant transparentes puisqu'elles ne font que révéler (au sens photographique) la façon dont le monument a impressionné (aux deux sens du terme) le visiteur. La médiation dans ce cas ne produit rien, elle n'est pas ce mouvement vers/de l'autre qu'avait décrit Élisabeth Caillet (1994), elle n'opère pas la rencontre, elle ne fait que préparer puis désigner le « déjà-là » et le «toujours-su » que notre rationalité avait occultés. 
21 Pour l'association Convergences, le pouvoir agissant du monument, du fait de ses caractéristiques énergétiques particulières, est susceptible d'opérer des transformations non seulement spirituelles mais physiques. Pour peu qu'il soit réceptif et un peu entraîné, le visiteur doit pouvoir ressentir les "énergies » dégagées par le lieu. Le stage se présente donc comme un apprentissage, une quête, un parcours initiatique qui permet d'abord de décrypter le symbolisme qui donne les clés d'une compréhension cachée puis d'en ressentir les effets. Dans ce cadre, le symbolisme doit être entendu comme un langage crypté, utilisé par les bâtisseurs pour transmettre un message, une vision du monde, les règles d'un parcours initiatique... Les décoder, c'est découvrir ce qu'ils peuvent nous apporter dans le monde d'aujourd'hui puisque les leçons des sages sont universelles et intemporelles, et les symboles toujours vivants.

On joue donc avec les images comme dans un rébus, on joue avec les mots en recourant à la « langue des oiseaux » : «Saint Hilaire parle d'un pot d'étain ; l'étain, c'est du sel d'étain; on fait des étincelles, il veut en fait parler de la révélation ${ }^{17}{ }^{17}$. L'exercice est quelque peu échevelé. Il ne repose que sur des jeux d'associations, le paradigme indiciaire étant exploité au maximum. On est dans un jeu de piste où toutes les interprétations sont bonnes, ou du moins sont valides pour celui qui les fait puisqu'elles ont un sens pour lui. Car « chaque symbole n'est que le reflet de nous-même ». On peut en effet décrire un symbole, le resituer dans un ensemble cohérent, comme une suite de chapiteaux, mais on ne peut en aucun cas présager de ce qu'il va signifier pour un individu donné, de la façon dont il va éclairer sa conscience. Le message d'un symbole n'est pas transmissible, il appartient à chacun de le vivre en fonction de sa propre expérience. Tout se recentre donc sur l'individu et ce que le symbole (et plus largement le stage) lui fait expérimenter.

23 Le programme est conçu comme une progression et une sorte de mise en condition pour recevoir, à l'issue de ces quatre jours, la « révélation », le « Graal ». À cet effet, il alterne des sessions de cours (en particulier sur la géométrie sacrée et ses références luni-solaires) et les circumambulations pédagogiques destinées à se remettre dans les pas des pèlerins ou à ressentir in situ les liens entre les différents lieux sacrés qui entourent la basilique. Il était demandé aux participants de jouer le jeu et de respecter les étapes d'une découverte progressive du site. Nous ne sommes ainsi entrés dans le narthex que le second jour du stage, dans la nef le troisième, tandis que la découverte de la crypte a été réservée au dernier jour, comme une sorte de parachèvement d'un parcours du plus lointain au plus intérieur.

24 Le premier jour nous a donc emmenés à quelques kilomètres de la colline, sur le site de fouilles archéologiques des Fontaines salées (commune de Saint-Père-sous-Vézelay), où l'on a dégagé les vestiges de thermes gallo-romains. Le but de la visite est de montrer que tout est relié dans le temps et l'espace. La vue, au loin, de la " colline du Scorpion », surmontée de la basilique, et la démonstration que les Fontaines salées s'inscrivent, comme elle, dans un quadrilatère dit " de Jérusalem » suffisent à établir ces liens. La présence à proximité du site d'une grosse pierre supposée être un vestige mégalithique vient encore prolonger leur profondeur temporelle. Les participants sont invités à s'asseoir sur la pierre pour ressentir les énergies qui s'y concentrent et ce qu'elle peut signifier. 


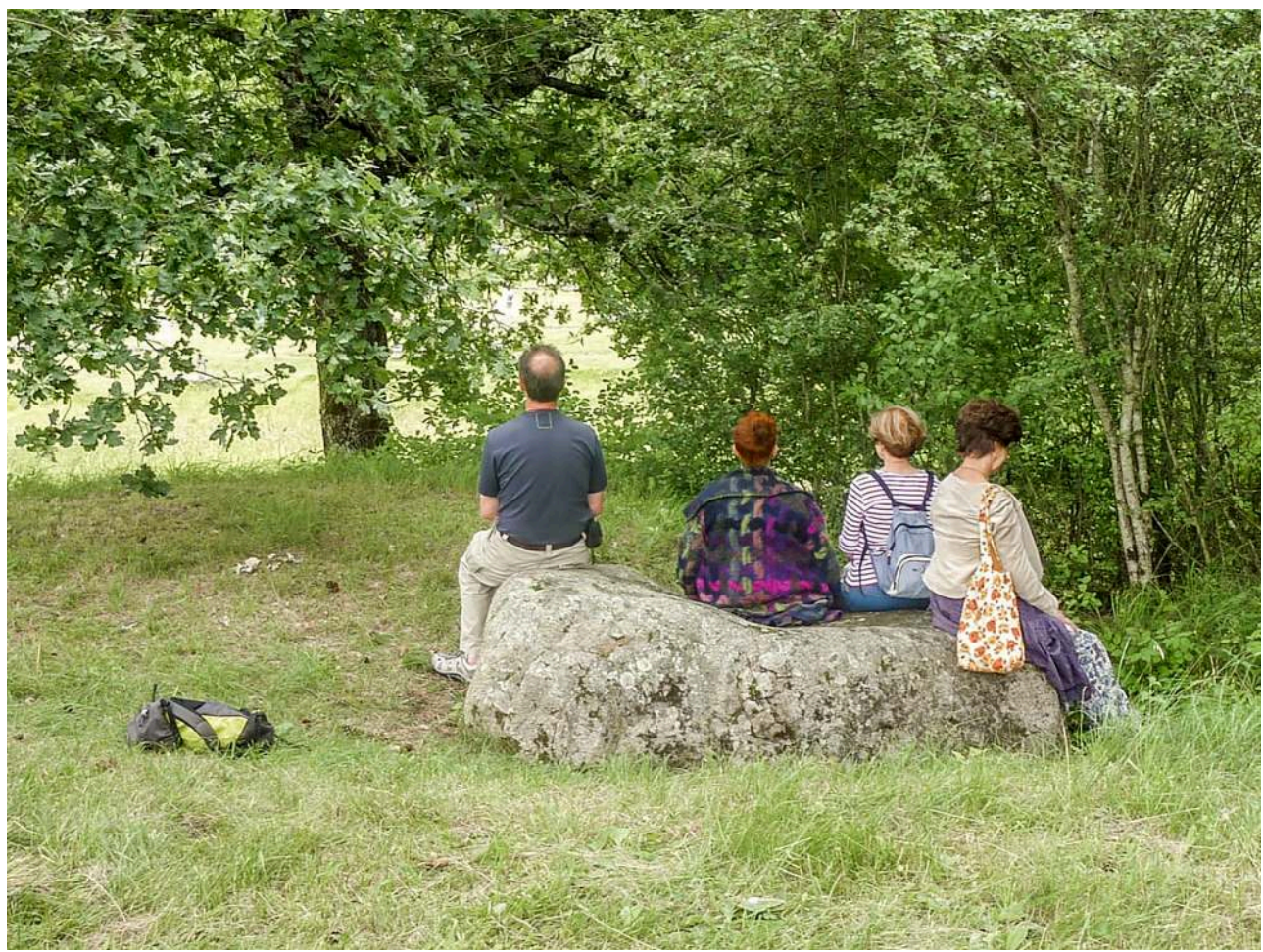

Assis sur un mégalithe, les stagiaires sont invités à ressentir les énergies telluriques. Comme l'indique la brochure du stage, «la connaissance théorique doit passer par l'expérience "charnelle" et s'inscrire dans le corps».

(c) Claudie Voisenat.

Le lendemain matin est consacré à la promenade du pèlerin. "Nous allons refaire le périple d'arrivée du pèlerin qui arrivait à Vézelay, selon la tradition. » Nous descendons donc par le rempart sud avant de remonter par la chapelle franciscaine de la Cordelle. Au pied de la colline, nous nous arrêtons à la fontaine de la Madeleine, où le pèlerin, nous dit-on, se lavait et se purifiait avant d'aborder la montée vers la basilique. Là encore, tout est lié et on nous montre que l'espace entre la margelle de la fontaine et le sommet de la voûte de pierre qui la surplombe fait exactement la taille de la « canne de Vézelay", mesure qui aurait servi d'étalon aux constructeurs de la basilique. En remontant vers le sommet de la colline, on s'arrête devant une croix plantée sur une grosse pierre, qui marque le lieu où saint Bernard aurait appelé à la croisade le 31 mars 1146. L'alliance de la croix et du mégalithe: nouvelle preuve que tout ne fait que s'associer, la pierre aurait été amenée de loin, à grand effort, pour sceller et symboliser la force de la rencontre entre la Terre-mère et le divin céleste, le passé païen et l'avenir chrétien.

Le cheminement du pèlerin nous conduit finalement jusqu'au narthex de la basilique où, nous explique-t-on, se trouve un carré magique, ou "carré de Saturne », délimité par quatre piliers, juste en face du portail royal. Le carré de Saturne est un carré de trois cases sur trois, dont le total des nombres le composant est égal à 15. «Le carré de Saturne est le moyen mathématique qui permet de faire la liaison avec l'énergétique d'un lieu. Sur le plan vibratoire, ça se manifeste par la création d'une grille géomagnétique naturelle sur laquelle le constructeur placera la porte du sanctuaire. Le narthex de Vézelay ou celui de Saint-Benoît-sur-Loire ne sont qu'une des applications 
symboliques de ce principe, et c'est la rotation des forces cosmiques tous les neuf ans qui donne le code de lecture des chapiteaux. " Là encore, nous devons exercer notre ressenti. Il faut nous placer sur le carré magique, nous y déplacer et trouver le chemin pour en sortir. Il n'existe normalement qu'un seul carré par lequel la sortie soit en harmonie avec le lieu et il change régulièrement selon la rotation des planètes. Nous devrions donc ressentir comme une sorte de frein, de barrière invisible, lorsque nous cherchons à sortir par un des autres carrés. Chacun jette un œil en coin sur ce que font les autres.

Le lendemain matin, nous mettons en application le cours de la veille sur la lecture luni-solaire de la basilique. Nous pénétrons donc dans la nef, mais pour le moment nous devons nous contenter d'arpenter les travées en passant de l'une à l'autre par la croisée du transept. La travée de gauche est la travée lunaire; elle reste le plus souvent dans l'ombre, même si la lumière vient, à un moment ou à un autre de l'année, en éclairer chacun des détails, jusqu'au basilic lové au pied du premier pilier. On nous explique que les chapiteaux de ce côté lunaire représentent la matière, sa maîtrise par le pouvoir temporel, la lutte avec nos démons intérieurs, la nécessité de faire des choix (combat de démons pour la capture d'une âme, jugement de sainte Eugénie, sacrifice d'Abraham...). Ce côté lunaire, c'est le chemin de l'apprenti. Nous traversons le transept pour parvenir à la travée solaire, celle de droite, qui se trouve beaucoup plus éclairée. Les chapiteaux, nous dit-on, y représentent la maîtrise de la matière par le pouvoir spirituel (lutte de Jacob avec l'ange, Daniel dans la fosse aux lions...), jusqu'à une sorte d'apothéose avec la conversion de saint Eustache, puis l'on nous présente les tentations qui marquent le retour dans le monde extérieur (la luxure ou la colère, le suicide de Judas...). La travée solaire représente le chemin du compagnon. De retour dans l'avant-nef, nous apprenons que le parcours que nous avons effectué est celui que devaient faire les pèlerins : un cheminement de régénération physique et spirituelle, de l'ombre à la lumière, de la matière à l'esprit. Celui-ci étant accompli, on est prêt à retourner affronter le monde extérieur, mais on peut aussi emprunter la voie du maître, celle qui traverse la nef en son milieu et arrive directement au jubé. C'est le troisième et avantdernier jour du stage et nous sommes maintenant dignes de retourner dans la nef en empruntant cette fois la voie réservée aux initiés, celle qui nous rend «maître des lieux ». On jette des regards torves à un groupe de touristes bruyants qui circulent à contresens. 


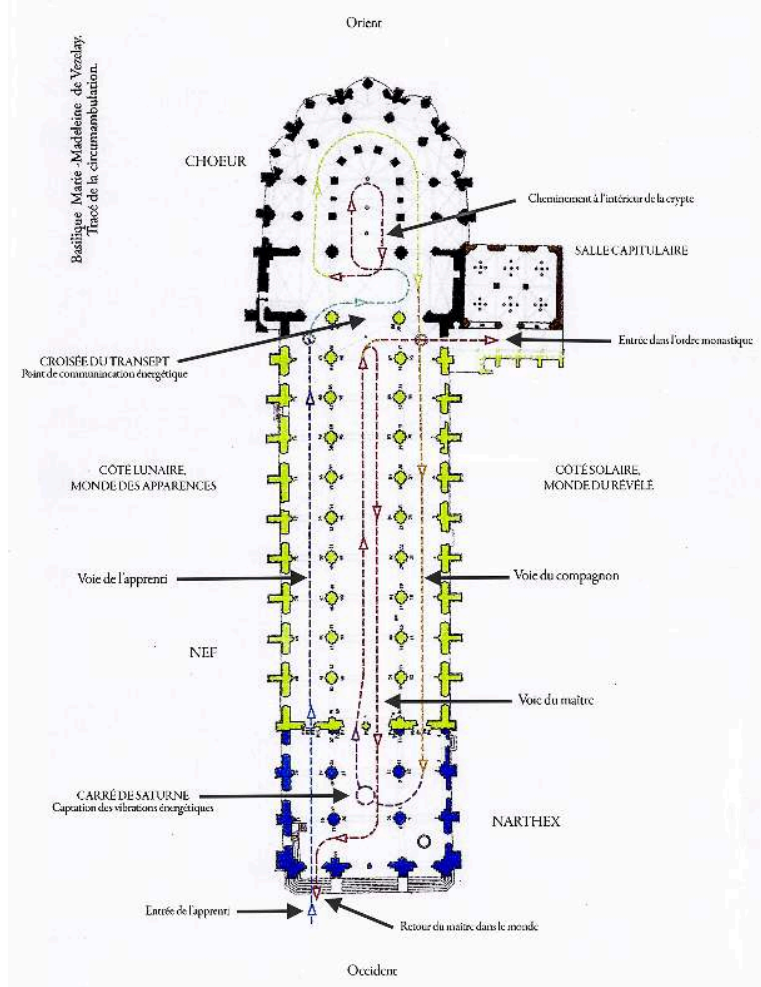

Les parcours dans la basilique, basés sur une lecture luni-solaire et sur les jeux de lumière à l'intérieur de l'édifice : les voies de l'apprenti, du compagnon et du maître ; le cheminement à l'intérieur de la crypte. Matériel de stage annoté par l'auteure.

(c) Claudie Voisenat.

L'après-midi est consacré à approfondir, en salle de cours, le système luni-solaire de la basilique et à comprendre la façon d'y cheminer pour en recueillir les enseignements. On nous explique également comment pénétrer et circuler dans la crypte. Il importe en effet d'y arriver par la travée lunaire de la nef, de traverser la croisée du transept qui est le point le plus énergétique de la basilique (celui où l'énergie cosmique captée par le clocher rencontre l'énergie tellurique), de rentrer dans la crypte par la porte qui se situe du côté solaire et d'en ressortir par la porte du côté lunaire. Un tel parcours doit nous amener à recroiser, dans la crypte, notre propre chemin. C'est d'ailleurs le but recherché : on nous explique en effet que l'être humain est polarisé (positif devant, négatif derrière pour l'homme; la polarité de la femme étant inversée, principe à l'origine de la Magia sexualis de Pascal Bewerly Randolph (1872), et que revenir sur nos pas nous permet d'effacer notre passage, l'empreinte énergétique que nous laissons, et de maintenir le lieu dans sa pureté originelle.

Munis de ces informations, nous apprenons que le lendemain matin nous aurons quartier libre afin que nous puissions parcourir la basilique à notre rythme et découvrir la crypte que nous n'avons encore jamais vue. On nous demande de rester discrets, de ne pas nous livrer à des rituels bizarres et de ne pas nous déchausser. Lors d'un stage précédent, une personne s'était déchaussée pour mieux sentir l'énergie tellurique de la crypte, tout le monde l'avait imitée et les sœurs responsables de la basilique avaient été fort mécontentes de trouver un amoncellement de chaussures à l'entrée. Selon l'intervenant, c'était de toute façon inutile, une énergie tellurique qui a traversé des kilomètres de croûte terrestre n'allait pas se laisser arrêter par un centimètre de 
semelle... Convaincu par le raisonnement, le groupe promet de ne pas finir en chaussettes. On nous demande aussi de ne pas prendre au pied de la lettre l'obligation de faire le tour de la crypte. Les sœurs ont en effet installé un tapis destiné à la prière tout au fond. Il faudra donc contourner le tapis et ne pas rester bloqués devant comme la personne que les maîtres de stage avaient un jour retrouvée en larmes, faute d'avoir pu suivre le chemin indiqué. On nous explique que les intervenants seront de toute façon à proximité au cas où quelqu'un ne se sentirait pas bien et aurait besoin d'être « recentré ». C'est que le moment était venu, semblait-il, de la grande révélation qu'on nous promettait depuis le départ. De fait, l'une des stagiaires repartit en larmes, frappée en sortant de la crypte par la vision de la croix grossièrement taillée que des prisonniers allemands avaient tenu à apporter en 1946 dans le cadre de la Croisade pour la paix qui avait marqué le $900^{\mathrm{e}}$ anniversaire de l'appel de Bernard de Clairvaux. Issue d'une famille de résistants, élevée dans le récit héroïque de la lutte contre l'envahisseur nazi, elle venait de comprendre "que les Allemands aussi avaient souffert ». Elle pleurait encore quand elle nous en parla lors de la réunion finale de partage d'expérience sur les apports du stage, où chacun fut convié à raconter son «ressenti » personnel. Les stagiaires étaient très émus : l'un déclara que le stage lui avait donné le sentiment de s'inscrire dans la lignée des hommes qui avaient œuvré à la construction de ces bâtiments et que cela permettait de ne pas désespérer de faire partie de l'humanité à une époque où l'on n'a pas quantité de raisons d'en être fier. Un couple se livra à une sorte de confession publique: la femme avouant avoir encore beaucoup de travail à faire sur sa vanité et son égoïsme; le mari s'accusant d'être encore trop cartésien bien que, depuis deux ans, la «croûte " se brisât peu à peu : le stage avait été pour lui un pas de plus vers le lâcher-prise. L'émotion était très forte, $\mathrm{y}$ compris pour l'un des intervenants qui, très empathique et les larmes aux yeux, expliqua combien ces stages l'enrichissaient et étaient pour lui des expériences humaines de très grande intensité. L'organisateur du stage, au contraire, très distancié et fidèle à son personnage, fermait la voie au pathos. Ainsi, lorsque la stagiaire évoqua en pleurant la croix des Allemands et alors que l'émotion était à son comble, il se mit à expliquer les circonstances et le déroulement de cette Croisade pour la paix de 1946, accumulant les informations factuelles pour dépassionner la situation et laisser à chacun le temps de se reprendre. La scène avait des allures de thérapie de groupe et, d'une certaine façon, le stage avait tenu ses promesses: le site patrimonial avait ramené chacun vers lui-même et le détour par la connaissance n'était finalement qu'un cheminement vers un développement personnel.

En conclusion et en guise de viatique, un intervenant nous dit: "Voilà, vous avez accompli un pèlerinage, pas au sens chrétien du terme, mais un pèlerinage tout de même. Maintenant, vous êtes reliés. On ne précise pas à quoi pour laisser les potentialités ouvertes, mais vous êtes reliés. » Il ajoute plus tard : «Vous êtes reliés et ça vous suivra toute votre vie. »

\section{Patrimoine et mise en capacité d'agir}

La visite au monument a ainsi, idéalement, permis de vivre une expérience transformatrice, cette voie de l'entrée en conscience, théorisée par Marylin Ferguson (1981) dans son ouvrage Les Enfants du Verseau. L'expérience transformatrice, que décrit Ferguson, peut advenir selon des modalités aussi diverses que les individus et leur 
biographie : accidents, maladies, deuils, rencontres, lectures, usage de psychotropes, méditation, etc., mais elle a toujours pour caractéristique d'ouvrir la voie à un nouveau pouvoir, celui de transformer la société. Cette expérience offre en effet à l'individu le pouvoir d'agir sur lui-même et, in fine, sur le monde, puisque le changement global est opéré par la somme des transformations individuelles. Le potentiel de changement ne réside donc plus dans le groupe, politique par exemple, mais dans chaque homme en particulier, qui doit opérer sa transformation personnelle. Le collectif n'est plus le cadre d'aucune sublimation ; seul l'individu peut changer le monde, ou plutôt l'addition « des gestes quotidiens des gens ordinaires ${ }^{18}$, cette " conspiration douce » qui « vise le retournement de conscience d'un nombre critique d'individus, suffisant pour provoquer un renouveau de la société » (Ferguson 1981:19) et qui réunit tous ceux qui ont la certitude spirituelle que tout peut être autrement en un puissant réseau de réseaux, sans centre ni dirigeants (ibid. : 220).

Pour l'ensemble des médiations observées, l'expérience et les connaissances acquises au cours de la visite doivent permettre au visiteur de reprendre sa route avec un nouvel élan, celle de son pèlerinage réel ou spirituel, et plus largement celle de sa vie, dans la mesure où toute vie est un pèlerinage. Pour la congrégation comme pour la Maison du visiteur, la beauté et son pouvoir d'élévation jouent un rôle essentiel dans ce don d'agentivité fait par le lieu à celui qui le visite. Pour la Fraternité de Jérusalem, spécialisée dans l'organisation de concerts, la musique, le chant et le son sont primordiaux. C'est ainsi qu'elle propose également des stages de violon dispensés par une moniale, sœur Colombe, violoniste professionnelle. Mais il lui est aussi arrivé d'offrir, à côté de sessions spirituelles, des stages de tapisserie haute-lisse, par exemple. La Maison du visiteur, quant à elle, nous en avons vu un exemple, complète son offre de prestations par des ateliers où le dessin, l'écriture ou encore la prise de parole, l'expression théâtrale, viennent faire dialoguer, à travers le stagiaire-visiteur, le XII et le $\mathrm{XXI}^{\mathrm{e}}$ siècles. Chacun est alors invité à se considérer comme " une pierre vivante de l'édifice humain » et à entrer en résonance avec une époque " qui représentait l'homme comme un être relié à la Terre, au cosmos et à Dieu ». Fidèle à son idée d'enseignement par l'expérience artistique, la directrice insiste sur le fait que les images sculptées par les tailleurs de pierre médiévaux peuvent agir sur les hommes d'aujourd'hui et que leur contemplation " peut bousculer certains conditionnements du regard et inspirer les créateurs d'images dans la recherche de cohérence entre éthique, technique et esthétique [...]. Actualiser [cet art], c'est entrer dans une interprétation re-créatrice de nos comportements et habitudes, un travail d'interprétation propice au surgissement de valeurs fondamentales dont notre société a un besoin vital $»^{19}$. On est bien dans l'idée que l'art, pour peu qu'il soit vivant, peut refaçonner le monde et ses valeurs.

Pour l'association Convergences, la connaissance théorique, celle des symboles et des tracés régulateurs, étant passée par l'expérience " charnelle » et s'étant inscrite dans le corps, de subtiles mais profondes transformations physiques et spirituelles, prenant la forme d'une véritable initiation, amènent les stagiaires à se sentir «reliés » et donc à être prêts à modifier leur vie et leur environnement. Mais le processus n'est pas plus explicité que cela, de sorte que la transformation et la mise en capacité d'agir sont en quelque façon performatives. On peut cependant noter que plusieurs participants exerçaient des métiers de producteurs de formes (architectes, graphistes, designers), qu'ils étaient venus dans l'attente de pouvoir réinjecter le savoir acquis dans leur pratique professionnelle et qu'ils semblaient certains à l'issue du stage que l'expérience 
qu'ils venaient de faire allait changer quelque chose à leur façon sinon d'exercer leur métier tout au moins de le vivre.

Jean-Claude Gadreau, photographie extraite de l'ouvrage de Françoise Leriche-Andrieu, Initiation à l'art roman, Zodiaque, coll. "Les travaux des mois », 1984.
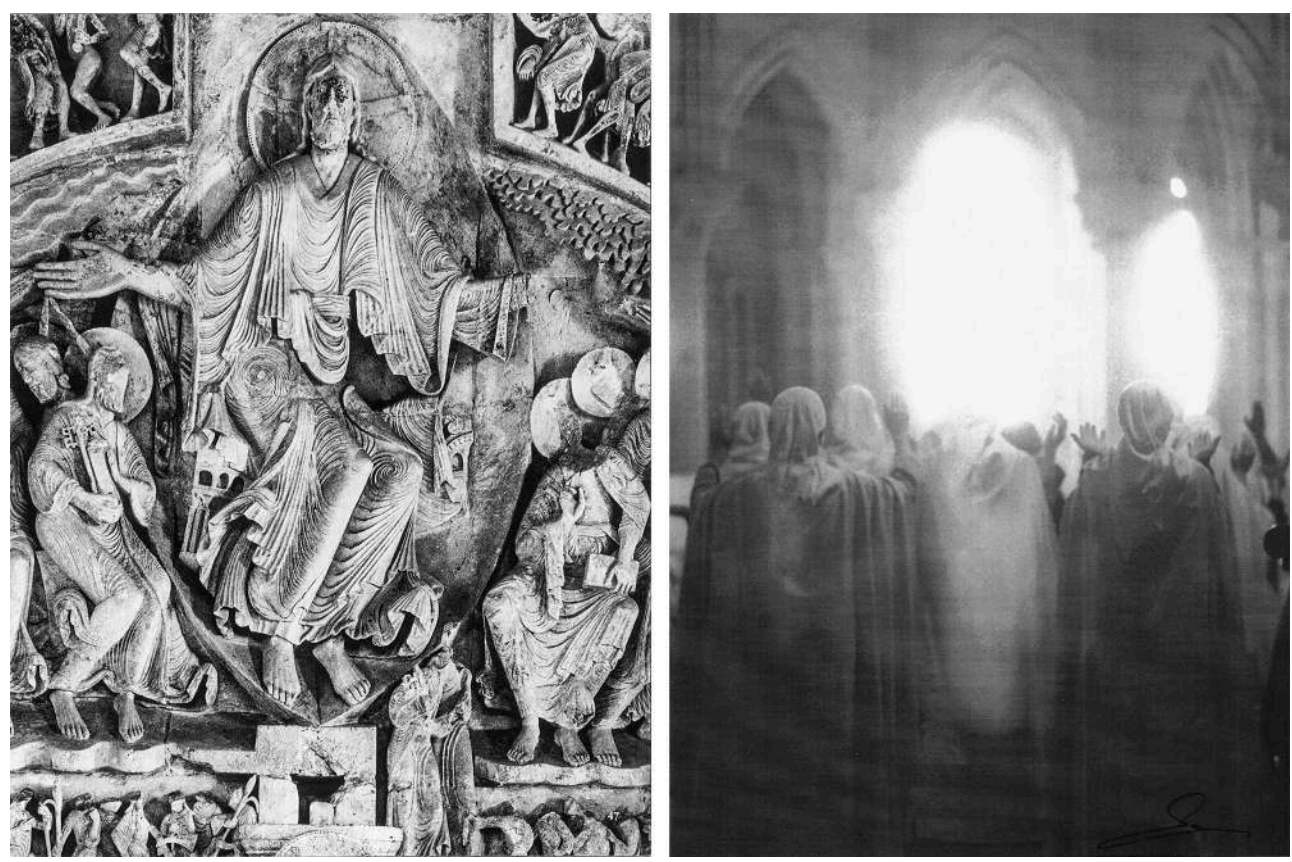

À partir du milieu des années 1950, les éditions Zodiaque animées par les moines de l'abbaye de la Pierre-Qui-Vire, ont popularisé une esthétique photographique de l'art roman, en noir et blanc, privilégiant le dépouillement et le traitement de la lumière. À la fin des années 1970, le père franciscain Hugues Delautre propose une lecture spirituelle des jeux de lumière dans la basilique. Une nouvelle forme d'esthétisation se met en place, où la clarté, l'éblouissement deviennent des métaphores visuelles de la révélation et de la grâce. On y retrouve à la fois le message chrétien de Saint Jean affirmant dans sa première épitre que Dieu est lumière et un symbolisme œcuménique susceptible d'être compris de tous, quelle que soit la religion.

(c) Jean-Claude Gadreau / éditions Zodiaque.

\section{Quand la médiation œuvre à son propre effacement} incidence sur les formes de médiations mises en œuvre. Dans ce cas précis où le patrimoine est en lui-même un médiateur entre les hommes d'aujourd'hui et ceux d'autrefois qui ont quelque chose à transmettre, la médiation devient, de fait, médiation d'une médiation et consiste essentiellement à mettre en scène son propre effacement. Elle doit en effet composer avec un paradoxe. Si le monument doit délivrer à chacun un message personnel et s'adresser à son intériorité, quelle est la place du médiateur dans ce dialogue intime? Si la rencontre doit déborder le cadre de la raison pour s'adresser à l'esprit, quel savoir nécessaire le médiateur doit-il transmettre pour permettre l'accès à la vérité du lieu?

Faute de temps, je n'aborderai pas ici les différentes stratégies mises en œuvre pour construire cette fiction, l'exemple cité plus haut de la rencontre dessinée puis révélée en donne une bonne illustration. Précisons seulement pour conclure que cet effacement du médiateur est au cœur de tout le dispositif de la Maison du visiteur. 
Celui-ci se place en effet sous le signe d'un dédoublement, d'une dissociation spatiale et temporelle de l'apprentissage et de la rencontre. La médiation se situe en amont de la visite proprement dite de la basilique. La Maison du visiteur, située sur la route qui mène au monument, propose en effet une "visite scénographique symbolique » avec des maquettes, des films, des reconstitutions de chapiteaux, etc destinés à donner un certain nombre d'informations factuelles pensées comme des "repères historiques, techniques et symboliques ", à aiguiser le regard et à attirer l'attention sur les jeux de l'ombre et de la lumière tels qu'ils ont été conçus par les bâtisseurs. Car si « les tailleurs d'images ont déposé dans le silence de la pierre d'humbles messages et de puissants enseignements [... qui] révèlent des questionnements universels $»^{20}$, nous avons aujourd'hui perdu les connaissances traditionnelles qui permettent de les lire. Nous avons aussi perdu la capacité d'observation, voire de contemplation qui leur permet de prendre existence et sens. Ce sont précisément ces compétences que la médiation entend restaurer. Une fois ce préalable accompli, le visiteur est prêt à aller voir la basilique, à y "cheminer selon son rythme ", seul, ouvert à la rencontre avec le lieu. La médiation proposée par la Maison du visiteur repose ainsi avant tout sur son propre effacement, et ce de façon parfaitement consciente, la directrice y insistera à maintes reprises. Elle ne manque d'ailleurs jamais d'évoquer cette caractéristique et même de la théoriser quand elle présente la structure : « Découvrir un patrimoine et plus encore le faire découvrir, c'est s'aventurer ensemble sur un sentier où le moindre détail devient l'indice d'une présence... Alors le visiteur se surprend à être lui-même visité... et le guide se tait pour laisser place à la rencontre $»^{21}$.

La dissociation entre l'initiation par la médiation et la rencontre initiatique que le visiteur doit accomplir avec le monument repose également sur un processus métonymique dans lequel la pierre vaut pour la basilique. C'est ce qui est mis en scène dans l'atelier "Lecture de chapiteaux", où les images taillées dans la pierre et reconstituées dans la cave de la Maison du visiteur entrent en dialogue avec chacun des participants. «La parole de pierre, c'est le chapiteau sculpté, la parole de chair, celui qui le regarde. De l'un à l'autre, des images courent, des mots se bousculent, des

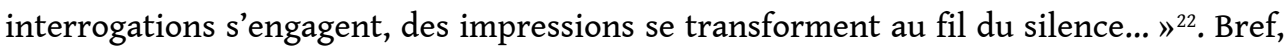
la rencontre s'opère, en dehors même de la monumentalité de la basilique, par la vertu et la force des images qu'elle contient et qui demeurent opératives même à distance et malgré le changement de support que représente un moulage en plâtre. Mais il n'y a là rien d'étonnant puisque la médiation revendique de se situer dans le monde des symboles, où les choses ont précisément pour valeur d'en représenter d'autres. 
Intérieur de la boutique du photographe Jean-Claude Gadreau à Vézelay.

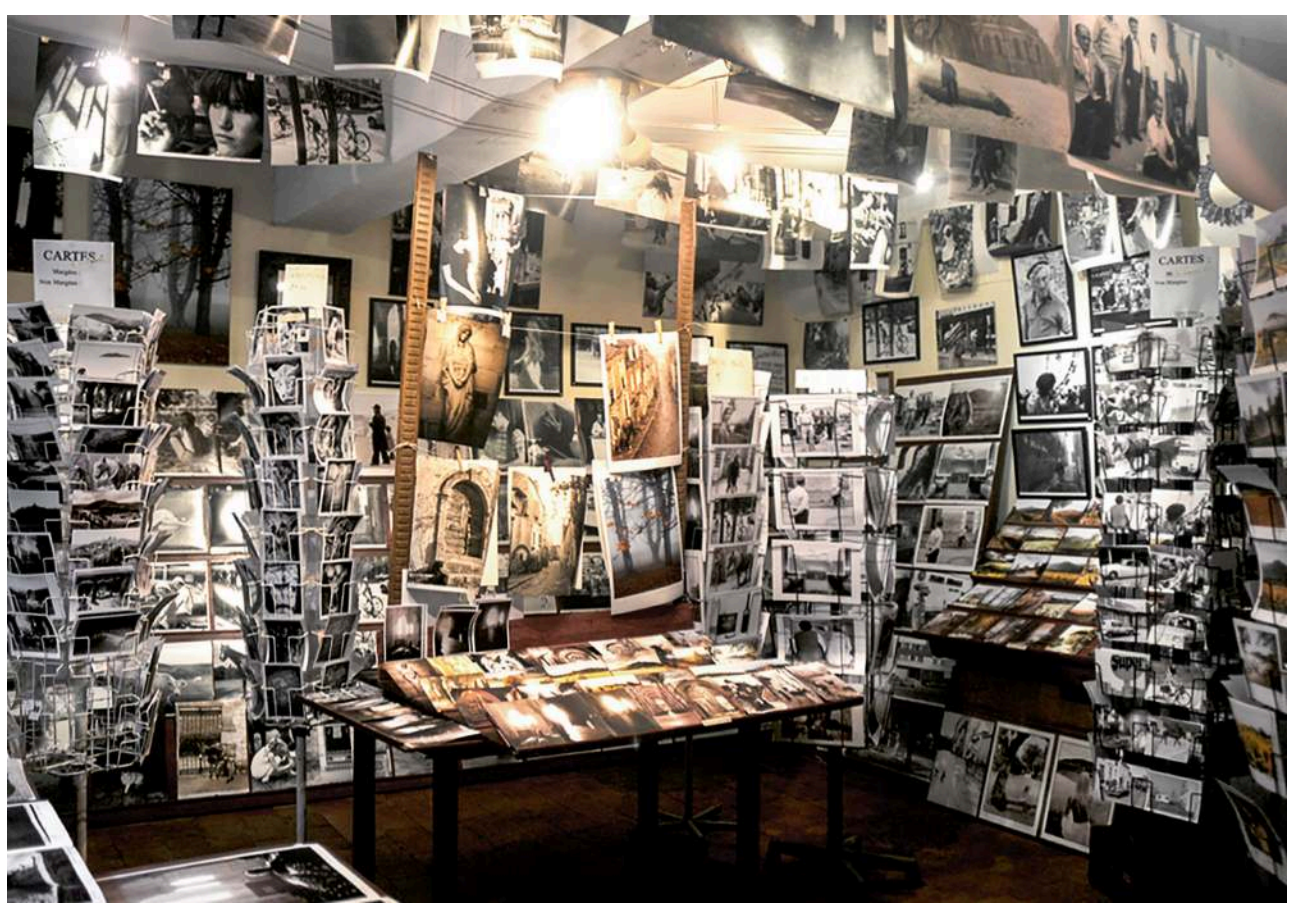

(C) Jean-Claude Gadreau.

Ces nouvelles formes de médiation du patrimoine, qui empruntent le langage de la spiritualité et du développement personnel, semblent mériter une attention particulière. Elles s'inscrivent en effet dans un continuum qui, partant des techniques de médiation les plus reconnues (le public comme acteur, l'expérience comme moyen de connaissance...), aboutit, à l'autre extrémité, à de véritables lectures ésotériques du patrimoine, où les sites sont pensés comme des lieux de ressourcement énergétique, leur caractère patrimonial n'étant que la conséquence (et la preuve) de caractéristiques vibratoires exceptionnelles. Autant de discours de sortie de l'histoire et d'entrée dans une nouvelle forme de naturalisme, «qui fait appel à des formes d'expérience corporéisées, extrêmement intimes, un discours de l'irrationalité du sujet », qui pose la question de l'existence aujourd'hui d'un « sens commun ", partagé, capable de dépasser ces nouvelles appropriations individuelles et intimes du patrimoine (Fabre 2016).

Participant d'un air du temps, s'adossant à un discours peu contestable qui remet l'individu au centre du savoir, ces nouvelles approches se révèlent extrêmement difficiles à analyser: sous leurs formes ésotériques les plus outrancières, elles ont tendance à susciter le rire et l'idée que personne ne peut les prendre au sérieux, hormis quelques hurluberlus de toute façon perdus pour la raison; sous leurs aspects les plus anodins, elles passent pratiquement inaperçues ou bien bénéficient d'un a priori positif au nom de la démocratisation du savoir.

Toutes, cependant, posent la question d'un changement de paradigme dans la perception $d u$ patrimoine. Toutes révèlent que l'un des ressorts du succès du patrimoine est sa capacité à émouvoir, mouvoir et transformer le public, qu'il est en cela, quelque restauré qu'il soit, un héritier de la méditation sur les ruines, qu'il partage, avec le spectacle des espaces sans bornes que propose parfois la nature, l'idée que l'homme participe de quelque chose qui le dépasse incommensurablement. Depuis son origine, le dispositif monumental ${ }^{23}$ porte la trace de son double parrainage, par les 
Lumières d'un côté et par le romantisme de l'autre. À travers les positions pour le moins contrastées d'un Alexandre Lenoir et d'un Quatremère de Quincy, se jouait déjà la tension entre le savoir et l'émotion, la théorie et l'expérience. Mais cette dualité intrinsèque des usages de ce que l'on appelle aujourd'hui le patrimoine prend actuellement un tour singulier. À mesure que s'estompe le pouvoir régalien, que les patrimoines (désormais pluriels) sont, bon an mal an, investis par des communautés (territoriales, sociales, religieuses...) au nom de valeurs qui leur sont propres, la grande geste nationale qui formait le support des savoirs patrimoniaux se délite, tandis que les attentes $\mathrm{du}$ public trouvent une véritable légitimité. Il en résulte d'étranges configurations, dont les médiations spirituelles ou ésotériques, sous leurs formes plus ou moins banalisées, sont l'une des manifestations. Même lorsqu'elles semblent au plus loin de la science, elles ne peuvent manquer d'interroger des professionnels du patrimoine, peu ou prou confrontés à l'injonction nouvelle de transmuer leurs savoirs historiques ou architecturaux en une expérience émotionnelle unique quoique partageable par le plus grand nombre.

Bien sûr, pareil sujet attire immanquablement les généralisations a priori : sur le retour d'un irrationnel qui menacerait les fondements mêmes de notre société démocratique, rationnelle et laïque ; ou qui, à l'inverse, viendrait réenchanter un monde épuisé par la prééminence de la matérialité en une sorte de retour du merveilleux refoulé; ou qui constituerait un "réensauvagement» de notre civilisation exsangue, balayée par le grand rire du paganisme et du tragique. Les interprétations ne manquent pas, largement relayées par les médias. Pour éviter cet écueil, la démarche ethnographique s'impose, d'autant que poser le problème en termes de rationalité et d'irrationalité est déjà biaiser l'analyse en se plaçant d'emblée dans le cadre d'un jugement de valeur. Ce sont en effet les rationalistes qui parlent d'irrationnel, opérant ainsi un nouveau " grand partage » entre ceux qui savent et ceux qui croient. Mais pour paraphraser Jean Pouillon qui parlait des ethnologues, «le rationaliste est un incroyant qui croit que le croyant croit». Le croyant, lui, pense qu'il sait, ou plutôt qu'il détient la connaissance. Or, la pratique anthropologique consiste à toujours faire crédit au groupe étudié d'une forme de rationalité qui lui est propre, à suspendre son incrédulité en quelque sorte. Il est indéniable en effet que, pour ceux qui y souscrivent, ces usages particuliers du patrimoine s'inscrivent dans une logique, dans le cadre d'un raisonnement, qu'ils sont étayés par des arguments, qu'ils déploient une vision cohérente des rapports entre le passé, le présent et l'avenir. Il est tout aussi indéniable que ces nouvelles façons de penser le patrimoine ont quelque chose à nous apprendre du monde dans lequel elles se déploient.

\section{BIBLIOGRAPHIE}

CAILLET Élisabeth, 1994, «L'ambiguïté de la médiation culturelle : entre savoir et présence », Publics et Musées, $\mathrm{n}^{\circ}$ 6, p. 53-73. Disponible en ligne, https://www.persee.fr/doc/ pumus_1164-5385_1994_num_6_1_1046 [lien valide en juin 2018]. 
DELAUTRE Hugues, 1979, Zodiaque, n 122, « Solstices à Vézelay », p. 1-16.

Dostö̈̈Esкi Fédor Mihailovič, 1993, L'Idiot, vol. 2, Arles, Actes Sud, coll. « Babel ».

DUBY Georges, 1976, Le Temps des cathédrales. L'art et la société (980-1420), Paris, Gallimard, coll. « Bibliothèque des histoires».

FABRE Daniel, 2013, Émotions patrimoniales, Paris, Éditions de la Maison des sciences de l'homme, coll. «Cahiers d'ethnologie de la France».

FABRE Daniel, 2015, « La Conquête de Vézelay », Paris, rapport pour le ministère de la Culture (département du Pilotage de la recherche et de la Politique scientifique).

FABRE Daniel, 2016, «L'ordinaire, le familier, l'intime, loin du monument », in HOTTIN Christian \& VOISENAT Claudie (dir.), Le Tournant patrimonial. Mutations contemporaines des métiers du patrimoine, Paris, Éditions de la Maison des sciences de l'homme, coll. « Cahiers d'ethnologie de la France », p. 43-58.

FABRE Daniel \& IUSo Anna (dir.), 2010, Les monuments sont habités, Paris, Éditions de la Maison des sciences de l'homme, coll. « Cahiers d'ethnologie de la France ».

FERGUSON Marilyn, 1981, Les Enfants du Verseau. Pour un nouveau paradigme, Paris, Calmann-Lévy.

FOUCAULT Michel (entretien avec Dominique Colas, Alain Grosrichard, Guy Le Gaufey, Jocelyne Levi, Gérard Miller, Judith Miller, Jacques-Alain Miller, Catherine Millot, Gérard Wajeman), 1994, « Le jeu de Michel Foucault », in FoucAuLT Michel, Dits et Écrits. 1954-1988, vol. 3, 1976-1979, Paris, Gallimard, coll. « Bibliothèque des sciences humaines », p. 298-329.

voISENAT Claudie \& LAGRANGe Pierre, 2005, L'Ésotérisme contemporain et ses lecteurs. Entre savoirs, croyances et fictions, Paris, Bibliothèque publique d'information / Centre Pompidou, coll. « Études et recherche » [en ligne] http://books.openedition.org/bibpompidou/640 [lien valide en juin 2018].

\section{NOTES}

1. Nef (1120-1140), narthex (1140-1150), chœur (1180-1210) pour remplacer le précédent dont la construction avait été achevée en 1104.

2. Site officiel de la basilique: http://www.basiliquedevezelay.org/\#!__site-partie-decouvrir/ vstc23=architecture (lien valide en juillet 2018). Cette dramaturgie lumineuse a été mise en évidence dans les années 1970, à la grande époque des Éditions Zodiaque et de l'esthétisation de l'art roman, par le père franciscain Hugues Delautre (1979).

3. Voir http://www.basiliquedevezelay.org/\#!__site-partie-decouvrir/vstc48=historique2 (lien valide en juillet 2018).

4. Voir http://www.lesamisdevezelay.fr/Site/index.php? option=com_content\&view=article\&id=69:un-present-multiforme-1950-a-nosjours\&catid=35:vezelay-dans-lhistoire\&Itemid=54 (lien valide en juillet 2018).

5. Le terrain s'est déroulé entre 2012 et 2015. Il a été fondé sur la participation systématique à des visites ou des stages organisés par trois acteurs majeurs de la scène vézelienne pour faire découvrir la basilique, le plus souvent à des adultes de passage, mais aussi, en quelques occasions, à un public scolaire adolescent. Ce travail d'observation a été complété par une analyse détaillée d'autres outils de médiation produits par ces acteurs : dépliants, affiches et surtout sites internet, qui prennent souvent la forme d'une médiation de la médiation et explicitent très utilement pour l'analyste les principes mis en œuvre dans les visites et les stages. Ce travail s'est déroulé en 
parallèle de celui de Daniel Fabre sur La Conquête de Vézelay (2015) qui mettait au jour les conflits de sacralité apparus avec la vocation littéraire et artistique du site. Il s'inscrit donc dans ce courant de l'anthropologie de l'instauration du patrimoine lancé au milieu des années 1990, par Daniel Fabre, au sein de la mission du Patrimoine ethnologique, au ministère de la Culture, puis au Laboratoire d'anthropologie et d'histoire de l'institution de la culture (Lahic) (voir entre autres Fabre 2010, 2013). Pour mes travaux précédents sur les lectures ésotériques, voir Voisenat (2005).

6. Ce nom reprend une phrase de Dostoïevski (1993 : 102) dans L'Idiot: "C'est vrai, prince, que vous avez dit, une fois : “C'est la beauté qui sauvera le monde” ?» demande Hippolyte Terentiev au prince Mychkine.

7. L'OCC a été, du début des années 1980 au milieu des années 1990, durant toute la durée du mandat socialiste, l'objet d'une vive polémique. Impliquée depuis le début des années 1970 dans l'animation socio-culturelle, elle est alors qualifiée de secte. On lui reproche d'«infiltrer le monde de l'enfance par la formule des stages et des séminaires dans le domaine des loisirs mais aussi du soutien scolaire et du développement culturel » et on lui retire son agrément Jeunesse et Sports. Voir le site officiel de l'occ qui donne sa version de l'affaire: http:// www.officeculturelcluny.org/controverses.php (lien valide en juillet 2018).

8. La présentation et les activités de la Maison du visiteur sont accessibles en ligne: http:// www.vezelay-visiteur.com/atelier-lecture-de-chapiteaux/ (lien valide en juillet 2018).

9. http://www.convergences-vezelay.com/ (lien valide en juillet 2018).

10. Les participants au stage formaient un petit groupe de vingt-sept personnes, dont une moitié à peu près était venue en couple (ou avec un·e ami·e) et dont l'âge moyen oscillait entre 40 et 50 ans. D'un milieu social plutôt aisé, sans doute déterminé par le prix du stage (près de 500 euros, sans compter l'hébergement et les repas du soir), une grande majorité des participants venait du milieu rural ou de petites villes de province. Hormis une Versaillaise, j'étais la seule à habiter la région parisienne. À noter également la présence de deux Suisses, qui d'ailleurs ne se connaissaient pas. Aucune donnée personnelle (l'âge, la profession) n'étant demandée au cours de la présentation, les informations sur la composition du groupe restent très succinctes. Pour autant qu'on puisse en juger d'après ce qu'ils livraient d'eux-mêmes, un bon nombre avaient fait des études supérieures (pilote de ligne, architecte, infographiste...) et tous venaient dans le cadre d'un questionnement personnel dont la simple énonciation pouvait mettre certains au bord des larmes, et dans le but avoué de se changer pour changer le monde et d'opérer, par les transformations sur soi-même et à petite échelle, une transformation de la société. On reconnaît là l'idée de la "conspiration douce » développée par Marilyn Ferguson dans son ouvrage Les Enfants du Verseau (1981), devenu une sorte de bible du Nouvel Âge (voir infra).

11. Il est intéressant de noter que le personnage de Marie-Madeleine, pourtant très en vogue au moment où je réalisais le terrain, n'y tient pratiquement aucune place et que les reliques y sont à peine évoquées, si ce n'est sur un plan historique pour expliquer les périodes de faste puis de déclin du monument.

12. Voir par exemple l'interprétation du chapiteau de la conversion de saint Eustache proposée par la Maison du visiteur et dont il est précisé dans le site de la congrégation que la partie qui représente le cerf est toujours éclairée.

13. https://vezelay-visiteur.com/christopher-kelly-ma-cambridge/, onglet: « La construction romane est un art inspiré, empirique et communautaire » (lien valide en juillet 2018).

14. La Maison du visiteur se présente en effet comme un centre d'interprétation qui, à l'aide de projections, de maquettes, de jeux de lumière, de reconstitutions de chapiteaux..., permet de préparer la visite à la basilique. Avant d'entrer dans les salles qui lui sont dédiées, la visite commence dans l'espace d'accueil, où l'on explique au groupe le sens universel de la spirale avant d'inviter l'un de ses membres, souvent un enfant, à pousser de la main, fermement appuyée au centre d'une spirale sculptée, le grand panneau de bois qui mène au cœur de la Maison. 
15. https://vezelay-visiteur.com/christopher-kelly-ma-cambridge/, onglet : «L'architecture romane offre une vision du monde, unifiée » (lien valide en juillet 2018).

16. Texte écrit pour la réception inaugurale de la Maison du visiteur le 25 mars 2003. Il poursuit : "Alors vient une autre question que nous éprouvons le besoin de nous poser plus particulièrement à nous-mêmes, animateurs du patrimoine. Cultivons-nous suffisamment ces témoignages d'art et de pensée avec la conscience que la foule des visiteurs d'aujourd'hui est une assemblée d'hommes en marche vers des terres intérieures? La mise en valeur et l'animation des patrimoines les plus prestigieux de l'humanité offrent-elles toujours aux hommes de toutes conditions, ces lieux d'intériorisation et de fraternisation? Sont-elles, en quelque sorte, des haltes d'incitation à un changement de regard et d'innovation sur le chemin de la Paix ?»

17. Sauf mention particulière, toutes les citations suivantes sont extraites des interventions ou explications des maîtres de stage ou des entretiens menés lors de l'enquête.

18. Selon les mots du magicien Gandalf dans le film Le Seigneur des anneaux : "Saroumane pense que seul un grand pouvoir peut tenir le mal en échec, mais ce n'est pas ce que j'ai découvert, je crois que ce sont les petites choses, les gestes quotidiens des gens ordinaires qui nous préservent du mal, de simples actes de bonté et d'amour» (extrait en ligne sous le titre: "Gandalf et Galadriel, la morale » : https://www.youtube.com/watch ?v =0gHtWV8Tsz0 [lien valide en juillet 2018]).

19. https://vezelay-visiteur.com/christopher-kelly-ma-cambridge/, onglets : « Le rôle de l'image et le rôle de la lumière» et "Placer le monde médiéval dans la perspective du monde contemporain » (lien valide en juillet 2018).

20. http://www.vezelay-visiteur.com/atelier-lecture-de-chapiteaux/ (lien valide en juillet 2018).

21. Présentation d'une conférence sur la Maison du visiteur in «Domaine de Chatressac. De la terre à la mer... ", programme en ligne de l'Atelier d'architecture et d'urbanisme (Atau), des Ateliers de Chatressac et de La Valenne, 2011: http://lucienherve.com/ PROGRAMME\%20WEB\%20DOMAINE\%20DE\%20CHATRESSAC\%202011\%20310K.pdf (lien valide en juillet 2018).

22. http://www.vezelay-visiteur.com/atelier-lecture-de-chapiteaux/, onglet: «Démarche de l'atelier Lecture de chapiteaux » (lien valide en juillet 2018).

23. Au sens foucaldien du terme " dispositif », tel que l'employait Daniel Fabre qui postulait, sans avoir eu le temps de parachever sa réflexion, que plusieurs dispositifs de valorisation des objets issus du passé se sont sédimentés au fil du temps: le trésor, l'art, le monument et enfin le patrimoine. Chaque dispositif étant, selon la définition de Michel Foucault (1994: 299), « un ensemble résolument hétérogène, comportant des discours, des institutions, des aménagements architecturaux, des décisions réglementaires, des lois, des mesures administratives, des énoncés scientifiques, des propositions philosophiques, morales, philanthropiques, bref : du dit aussi bien que du non-dit», il importait pour Daniel Fabre de bien cerner la chronologie, les caractéristiques et les apports de chacun de ces éléments.

\section{RÉSUMÉS}

De la cathédrale à l'ermitage de campagne, du château cathare au site mégalithique, des thermes gallo-romains aux abris sous roche, certains sites, très différents par leur nature, leur taille ou leur notoriété mais tous supposés entretenir un certain rapport avec le « sacré » donnent lieu à des visites et des guidages singuliers. Selon les médiateurs de ces usages inédits du patrimoine, ils 
ne sauraient être abordés à la seule lumière de la raison. Bénéficier du pouvoir de ces lieux où l'homme se sent « relié » au tout de l'univers impose de renoncer à son « mental », d'écouter son «moi intérieur », de "lâcher prise ». Toutes ces postures nécessitent un apprentissage qui est souvent le but même de la médiation. Le patrimoine, bien collectif par excellence, serait-il en passe de se transformer en instrument individuel de développement personnel? Privilégier le « ressenti ", l'expérience individuelle, le dialogue intérieur avec le monument, n'amène-t-il pas à délaisser des formes plus traditionnelles de communication d'un savoir lié à l'histoire de l'art et de la nation? Autant de questions posées par l'observation et l'analyse des médiations du patrimoine organisées autour d'un des fleurons du patrimoine religieux français, considéré comme un haut lieu de spiritualité : la basilique Sainte-Marie-Madeleine de Vézelay.

\section{INDEX}

Mots-clés : monuments, spiritualité, médiation, ésotérisme, Vézelay, individualisme

Keywords : monuments, spirituality, mediation, esoterism, Vézelay, individualism

\section{AUTEUR}

\section{CLAUDIE VOISENAT}

Chargée de recherche CNRS en ethnologie, membre du Laboratoire d'anthropologie et d'histoire de l'institution de la culture (Lahic, IIAC-CNRS). 